\title{
Exponential Stabilization of an Underactuated Autonomous Surface Vessel*
}

\author{
MAHMUT REYHANOGLU $†$
}

Key Words-Asymptotic stability; exponential convergence; discontinuous feedback control.

\begin{abstract}
This paper studies the problem of controlling the planar position and orientation of an autonomous surface vessel using two independent thrusters. It is first shown that although the system is not asymptotically stabilizable to a given configuration using a time-invariant continuous feedback, it is strongly accessible and small-time locally controllable at any equilibrium. Time-invariant discontinuous feedback laws are then constructed to asymptotically stabilize the system to the desired configuration with exponential convergence rates. A simulation example is included to demonstrate the results. (C) 1997 Elsevier Science Ltd.
\end{abstract}

\section{Introduction}

In the past few years, there has been a considerable amount of interest in the control of underactuated mechanical systems, i.e. systems with fewer inputs than degrees of freedom. The possibility of controlling a system with fewer than the typical number of actuators is indeed appealing, for it allows to reduce cost, weight as well as the occurrence of component failures. However, in general, underactuated systems present challenges which are not found in systems with full control. Controllability, for instance, which is usually implied in systems with full control, is not easy to determine in an underactuated system. Control synthesis for an underactuated system is also more complex than it is for a system with full control. While many interesting techniques and results have been presented for underactuated systems (Oriolo and Nakamura, 1991; Leonard, 1995; Egeland et al., 1996 Reyhanoglu et al., 1996; Spong, 1996), the control of these systems still remains an open problem.

This paper considers the problem of controlling the planar position and orientation of an autonomous surface vessel (marine vehicle) using two independent thrusters. The dynamics of this underactuated system is complex enough to yield a rich source of control problems, yet simple enough to permit a complete mathematical analysis. It has been shown (Wichlund et al., 1995) that underactuated vehicles do not satisfy Brockett's necessary condition (Brockett, 1983) if the unactuated dynamics contain no gravitational field component and, hence, in this case, these vehicles are not asymptotically stabilizable to a desired equilibrium solution using time-invariant continuous feedback laws. In this paper, it is first shown that although the system is not asymptotically stabilizable to a given equilibrium solution using a time-invariant continuous feedback, it is strongly accessible and small-time locally controllable at any equilibrium and, hence, the system is asymptotically stabilizable to a desired equilibrium using time-invariant discontinuous feedback laws. Discontinuous feedback laws are then constructed to

*Received 23 July 1996; revised 8 May 1997; received in final form 1 July 1997. Expanded version of a talk presented at the 15 th Benelux Meeting on Systems and Control, Mierlo, Netherlands, March 1996. Corresponding author Dr. M. Reyhanoglu. Automation/Robotics Technology, TSTC, Sweetwater, Texas 79556, U.S.A. Tel. 0019152357346 ; Fax 0019152357416 ; E-mailmreyhan@sweetwater.tstc.edu.

$\dagger$ Department of Applied Mathematics, Twente University of Technology, The Netherlands. achieve asymptotic stabilization. The methodology followed in the construction of the discontinuous fecdback laws is based on first transforming the system into a discontinuous one in which the design of feedback laws is easily carried out. Then, transforming back into the original coordinates yields discontinuous feedback laws which asymptotically stabilize the original system with exponential convergence rates. The discontinuous coordinate transformation employed here constitutes an example of a $\sigma$-process (Arnold, 1983), which has proved useful in the stabilization of a special class of nonholonomic systems (Astolfi, 1995). A preliminary version of this paper can be found in Reyhanoglu (1996).

The organization of this paper is as follows. In Section 2, the nonlinear control system describing the dynamics of a surface vessel with two independent thrusters is introduced. Controllability and stabilizability results are presented in Section 3. Discontinuous feedback laws are derived in Section 4. In Section 5 a simulation example is included. Finally, Section 6 consists of a summary of the main results and concluding remarks about future research.

\section{Mathematical model}

Consider the problem of controlling the Cartesian position and orientation of a surface vessel with two independent propellers as shown in Fig. 1. The kinematic model which describes the geometrical relationship between the earth-fixed (I-frame) and the vehicle-fixed (B-frame) motion is given as

$$
\begin{gathered}
\dot{x}=v_{x} \cos \psi-v_{y} \sin \psi, \\
\dot{y}=v_{x} \sin \psi+v_{y} \cos \psi, \\
\dot{\psi}=\omega_{z},
\end{gathered}
$$

where $(x, y)$ denotes the I-frame position of the center of mass of the vehicle, $\psi$ denotes the orientation angle; $\left(v_{x}, v_{y}\right)$ and $\omega_{z}$ are the linear and angular velocities of the vehicle in the B-frame. For simplicity the origin of the B-frame is assumed to be located at the center of the mass of the vehicle. It is also assumed that the vehicle is neutrally buoyant. Then the dynamic equations of motion of the vehicle can be expressed in the B-frame as

$$
M \dot{v}+C(v) v+D(v) v=\tau,
$$

where $v=\left(v_{x}, v_{y}, \omega_{z}\right)^{\mathrm{T}}$ denotes the velocity vector, $\tau=\left(F_{x}, 0, T_{z}\right)^{\mathrm{T}}$ denotes the vector of external force and torque generated by the two propellers. $M \in \mathbb{R}^{3 \times 3}$ is the inertia matrix, including hydrodynamic added mass; and $C(v) \in \mathbb{R}^{3 \times 3}$ and $D(v) \in \mathbb{R}^{3 \times 3}$ denote the Coriolis/centrifugal and the damping matrices, also including hydrodynamic added mass effects, respectively. The reader is referred to Fossen (1994) for the relevant concepts and the detailed formulation of the dynamics of marine vehicles. The following simplified model can be obtained by assuming that both the inertia matrix $M$ and the damping matrix $D$ are constant and diagonal:

$$
\begin{gathered}
m_{11} \dot{v}_{x}-m_{22} v_{y} \omega_{z}+d_{11} v_{x}=F_{x}, \\
m_{22} \dot{v}_{y}+m_{11} v_{x} \omega_{z}+d_{22} v_{y}=0, \\
m_{33} \dot{\omega}_{z}+\left(m_{22}-m_{11}\right) v_{x} v_{y}+d_{33} \omega_{z}=T_{z},
\end{gathered}
$$

where $m_{i i}, d_{i i}, i=1,2,3$, are positive constants. 


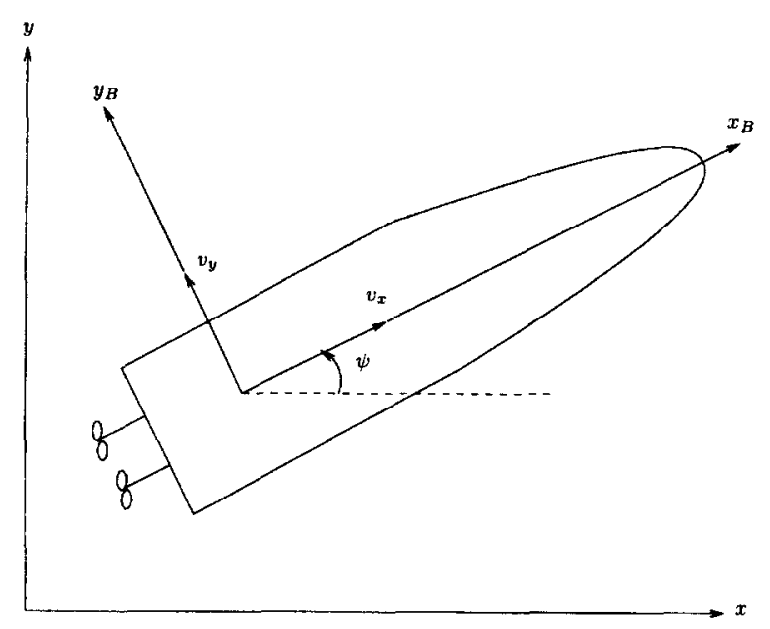

Fig. 1. Model of a surface vessel with two propellers.

Remark 1. Equation (6) can be rewritten in earth-fixed coordinates as

$$
\begin{gathered}
\mathrm{m}_{22}(\ddot{x} \sin \psi-\ddot{y} \cos \psi)+\left(m_{22}-m_{11}\right) \dot{\psi}(\dot{x} \cos \psi+\dot{y} \sin \psi) \\
+d_{22}(\dot{x} \sin \psi-\dot{y} \cos \psi)=0 .
\end{gathered}
$$

This equation represents a nonintegrable relation involving not only the generalized coordinates and velocities but also the generalized accelerations and, hence, it can be viewed as a second-order nonholonomic constraint. In contrast to the firstorder nonholonomic case (Bloch et al., 1992), a second-order nonholonomic constraint does not reduce the dimension of the state space. A set of three independent configuration variables and three velocity variables is required to completely specify the state of the system.

Remark 2. The surface vessel considered in this paper has no side thruster, i.e., $F_{y}=0$, but the controllability analysis and control synthesis of this paper can be easily extended to the cases where $\tau=\left(F_{x}, F_{y}, 0\right)^{\mathrm{T}}$ or $\tau=\left(0, F_{y}, T_{z}\right)^{\mathrm{T}}$. Note also that, for simplicity, both the inertia matrix $M$ and the damping matrix $D$ have been assumed to be diagonally dominant and constant. This assumption is satisfied, for instance, when (1) the vehicle body has two perpendicular axes of symmetry (e.g. an elliptic body), (2) hydrodynamic damping terms of order higher than one are negligible, and (3) changes in inertia are negligible. The subsequent development of this paper can be modified to account for more complex cases.

\section{Define the state variables}

$$
\begin{aligned}
\left(x_{1}, x_{2}, x_{3}, x_{4}, x_{5}, x_{6}\right)= & (\psi, x \cos \psi+y \sin \psi, \\
& \left.-x \sin \psi+y \cos \psi, v_{y}, \omega_{z}, v_{x}\right)
\end{aligned}
$$

so that the state equations are given by

$$
\begin{gathered}
\dot{x}_{1}=x_{5}, \\
\dot{x}_{2}=x_{6}+x_{3} x_{5}, \\
\dot{x}_{3}=x_{4}-x_{2} x_{5}, \\
\dot{x}_{4}=-\alpha x_{4}-\beta x_{5} x_{6}, \\
\dot{x}_{5}=u_{1}, \\
\dot{x}_{6}=u_{2},
\end{gathered}
$$

where $\alpha=d_{22} / m_{22}, \beta=m_{11} / m_{22}$ and

$$
\begin{gathered}
u_{1}=\left(T_{z}-d_{33} \omega_{z}+\left(m_{11}-m_{22}\right) v_{x} v_{y}\right) / m_{33}, \\
u_{2}=\left(F_{x}+m_{22} v_{y} \omega_{z}-d_{11} v_{x}\right) / m_{11} .
\end{gathered}
$$

Note that the variables $\left(x_{1}, x_{2}, x_{3}\right)$ correspond to the orientation angle of the vehicle and the B-frame coordinates of the center of mass of the vehicle, which parameterize the three-dimensional configuration space of the vehicle; and the variables $\left(x_{4}, x_{5}, x_{6}\right)$ correspond to the B-frame velocity components.

In the next section, we will consider the system [equations (8)-(13)] and study its controllability and stabilizability properties.

3. Controllability and stabilizability results form

Equations (8) $(13)$ define a nonlinear control system of the

$$
\dot{x}-f(x)+\sum_{i=1}^{2} g_{i}(x) u_{i},
$$

where $x=\left(x_{1}, x_{2}, x_{3}, x_{4}, x_{5}, x_{6}\right)^{\mathrm{T}} \in \mathbf{M}=S \times R^{5}$ is the state, and $f$ and $g_{i}, i=1,2$, are the drift and control vector fields given by

$$
\begin{gathered}
f=x_{5} \frac{\partial}{\partial x_{1}}+\left(x_{6}+x_{3} x_{5}\right) \frac{\partial}{\partial x_{2}}+\left(x_{4}-x_{2} x_{5}\right) \frac{\partial}{\partial x_{3}} \\
-\left(\alpha x_{4}+\beta x_{5} x_{6}\right) \frac{\partial}{\partial x_{4}}, \\
g_{1}=\frac{\partial}{\partial x_{5}}, \quad g_{2}=\frac{\partial}{\partial x_{6}} .
\end{gathered}
$$

Note that the set of equilibrium solutions corresponding to $u=0$ is given by the equilibrium manifold

$$
\mathbf{M}^{e}=\left\{x \in \mathbf{M} \mid x_{4}=x_{5}=x_{6}=0\right\} \text {. }
$$

It is easily verified that the linearization of equations (8)-(13) about an equilibrium $x^{c}$ has an uncontrollable eigenvalue at the origin. This implies that a nonlinear analysis is necessary in order to characterize the controllability and stabilizability properties of the system. Note that since the linearization of equations (8)-(13) is not stabilizable, the system cannot be exponentially stabilized at an equilibrium using smooth feedback (Zabczyk, 1989). Moreover, it is easy to see that the system does not satisfy Brockett's necessary condition (Brockett, 1983) and, hence, it is not asymptotically stabilizable to a desired equilibrium solution using time-invariant continuous feedback.

We now consider the nonlinear control system [equations (8) - (13)] and employ certain results of nonlinear control theory. We refer the reader to Nijmeijer and van der Schaft (1990) and Sussmann (1987) for the relevant controllability definitions and results used in the subsequent development.

The following results characterize the controllability and stabilizability properties of the underactuated vehicle dynamics described by equations $(8)-(13)$.

Proposition 1. The underactuated vehicle dynamics described by equations (8)-(13) is strongly accessible on $\mathbf{M}$.

Proof. Consider the system [equations (8)-(13)]. Since the vector fields

$$
g_{1}, g_{2},\left[f, g_{1}\right],\left[f, g_{2}\right],\left[g_{2},\left[f, g_{1}\right]\right],\left[\left[f, g_{2}\right],\left[f, g_{1}\right]\right]
$$

span a six-dimensional space at any point $x \in \mathbf{M}$, the strong accessibility Lie algebra rank condition is satisfied at any point. Hence, the system is strongly accessible on $\mathbf{M}$.

Proposition 2. The underactuated vehicle dynamics described by equations $(8)-(13)$ is small-time locally controllable at any equilibrium $x^{e} \in \mathbf{M}^{e}$.

Proof. Consider the system [equations(8)-(13)]. Now, following Sussmann (1987), let $\operatorname{Br}(X)$ denote the smallest Lie algebra of vector fields containing $f, g_{1}$, and $g_{2}$ and let $B$ denote any bracket in $\operatorname{Br}(X)$. Let $\delta^{0}(B), \delta^{1}(B)$ and $\delta^{2}(B)$ denote the number of times $f, g_{1}$ and $g_{2}$, respectively, occur in the bracket $B$. The degree of $B$ is equal to the value of $\sum_{i=0}^{2} \delta^{i}(B)$. The Sussmann condition for small-time local controllability is essentially that the so-called bad brackets, the brackets with $\delta^{0}(B)$ odd and $\delta^{1}(B), \delta^{2}(B)$ even, must be a linear combination of good (i.e. not 
of the bad type) brackets of lower degree at the equilibrium. The degree of a bad bracket must necessarily be odd. The only bad bracket of degree one is $f$ which vanishes at any equilibrium. The bad brackets of degree three are brackets with $\delta^{0}=1$ and $\delta^{i}=2, i=1$ or 2 , and all are identically zero vector fields. It follows that the Sussmann condition is satisfied at $x^{\mathrm{e}}$. Hence, the system is small-time locally controllable at $x^{\mathrm{e}}$.

Since the system is real analytic, the above controllability results imply the existence of piecewise analytic feedback laws (Sussmann, 1979) which asymptotically stabilize the closed-loop system to a given $x^{e}$. In the next section, guided by these results, we will focus on designing asymptotically stabilizing discontinuous feedback laws for the systern.

Remark 3. Note that in the absence of damping, after a variable reordering, equations (8)(13) take the form of those used by Coron and Kerai (1996) for the construction of locally stabilizing time-varying almost-continuous feedback laws for a rigid spacecraft with only two control torques. These time-varying feedback laws can be used, with minor modifications, to locally asymptotically stabilize the system [equations (8)-(13)]. It is also possible to use geometric phase ideas which proved useful for stabilizing mechanical systems with first-order nonholonomic constraints (Bloch et al., 1992; Reyhanoglu and McClamroch, 1992). It should be remarked that the geometric phase approach yields discontinuous feedback laws with many control switchings. In this paper, we will consider construction of discontinuous feedback laws which yield global asymptotic stabilization with at most one switching.

\section{Discontinuous feedback control laws}

In this section, we will consider the problem of designing feedback control laws of the form $u=u(x)$ for the system [equations (8)-(13)]. As discussed in the previous section, the system cannot be asymptotically stabilized to an equilibrium using any time-invariant continuous feedback. Therefore, we restrict our consideration to designing time-invariant discontinuous feedback laws.

Note that the problem of stabilizing the system to a given equilibrium $x^{\mathrm{e}} \in \mathbf{M}^{\mathfrak{t}}$ can be reduced to the problem of stabilizing the system to the origin via an appropriate state transformation. Hence, without loss of generality, we focus only on the problem of feedback stabilization to the origin, i.e. $x^{\mathfrak{c}}=0$.

We will first study the problem of stabilizing the following reduced-order system, which is obtained by considering the subsystem [equations (8)-(11)] and letting $\left(x_{5}, x_{6}\right)$ be the control variables $\left(v_{1}, v_{2}\right)$ :

$$
\begin{gathered}
\dot{x}_{1}=v_{1}, \\
\dot{x}_{2}=v_{2}+x_{3} v_{1}, \\
\dot{x}_{3}=x_{4}-x_{2} v_{1}, \\
\dot{x}_{4}=-\alpha x_{4}-\beta v_{1} v_{2} .
\end{gathered}
$$

4.1. Stabilization of the reduced system. The idea that will be employed is based on first transforming the reduced system [equations $(17)-(20)]$ into a discontinuous one by applying a discontinuous coordinate transformation, e.g. by applying a $\sigma$ process (see Arnold, 1983). From the analytical point of view, the $\sigma$-process, also termed as the process of resolution of singularities, consists of a rational coordinate transformation.

Consider the reduced system [equations $(17)-(20)]$. Restricting consideration to $x_{1} \neq 0$, apply the $\sigma$-process

to oblain

$$
y=x_{1}, \quad z_{1}=x_{2}, \quad z_{2}=\frac{x_{3}}{x_{1}}, \quad z_{3}=\frac{x_{4}}{x_{1}}
$$

$$
\begin{gathered}
\dot{y}=v_{1}, \\
\dot{z}_{1}=v_{2}+y z_{2} v_{1}, \\
\dot{z}_{2}=z_{3}-\frac{z_{1}+z_{2}}{y} v_{1}, \\
\dot{z}_{3}=-\alpha z_{3}-\frac{z_{3}+\beta v_{2}}{y} v_{1} .
\end{gathered}
$$

The feedback control law

$$
\begin{aligned}
& v_{1}=-k y, \\
& v_{2}=-l z,
\end{aligned}
$$

where $z=\left(z_{1}, z_{2}, z_{3}\right)^{\mathrm{T}}$, and $k>0$ and $l=\left(\begin{array}{lll}l_{1} & l_{2} & l_{3}\end{array}\right)$ are the gains, yields the reduced closed-loop system

$$
\begin{gathered}
\dot{y}=-k y, \\
\dot{z}_{1}=-l_{1} z_{1}-l_{2} z_{2}-l_{3} z_{3}-k y^{2} z_{2}, \\
\dot{z}_{2}=k z_{1}+k z_{2}+z_{3}, \\
\dot{z}_{3}=-k \beta l_{1} z_{1}-k \beta l_{2} z_{2}+\left(k-\alpha-k \beta l_{3}\right) z_{3} .
\end{gathered}
$$

The $z$-dynamics can be rewritten as

$$
\dot{z}=\left(A_{1}+A_{2}(t)\right) z,
$$

where

$$
\begin{gathered}
A_{1}=\left(\begin{array}{ccc}
-l_{1} & -l_{2} & -l_{3} \\
k & k & 1 \\
-k \beta l_{1} & -k \beta l_{2} & k-x-k \beta l_{3}
\end{array}\right), \\
A_{2}(t)=\left(\begin{array}{ccc}
0 & -k y_{0}^{2} \mathrm{e}^{-2 k t} & 0 \\
0 & 0 & 0 \\
0 & 0 & 0
\end{array}\right) .
\end{gathered}
$$

It can be easily seen that if $k \neq \alpha$, the spectrum of the matrix $A_{1}$ can be assigned arbitrarily through the gain matrix $l$. Clearly, the $y$-dynamics is globally exponentially stable at $y=0$. Moreover, since the matrix $A_{2}(t)$ given by equation (33) goes to zero as $t \rightarrow \infty$ and

$$
\int_{0}^{\infty}\left\|A_{2}(t)\right\| \mathrm{d} t<\infty,
$$

the $z$-dynamics can also be rendered globally exponentially stable at the origin $z=0$ by selecting $l=\left(\begin{array}{lll}l_{1} & l_{2} & l_{3}\end{array}\right)$ such that the matrix $A_{1}$ given by equation (32) is a Hurwitz matrix (see Slotine and $\mathrm{Li}, 1991$, Section 4.2.2).

Note that in the $\left(x_{1}, x_{2}, x_{3}, x_{4}\right)$ coordinates the controls [equations (25) and (26)] take the form

$$
\begin{gathered}
v_{1}\left(x_{1}, x_{2}, x_{3}, x_{4}\right)=-k x_{1}, \\
v_{2}\left(x_{1}, x_{2}, x_{3}, x_{4}\right)=-l_{1} x_{2}-l_{2} \frac{x_{3}}{x_{1}}-l_{3} \frac{x_{4}}{x_{1}}
\end{gathered}
$$

and the reduced closed-loop system is given by

$$
\begin{gathered}
\dot{x}_{1}=-k x_{1}, \\
\dot{x}_{2}=-l_{1} x_{2}-l_{2} \frac{x_{3}}{x_{1}}-l_{3} \frac{x_{4}}{x_{1}}-k x_{1} x_{3}, \\
\dot{x}_{3}=x_{4}+k x_{1} x_{2}, \\
\dot{x}_{4}=-k \beta l_{2} x_{3}-\left(k \beta l_{3}+\alpha\right) x_{4}-k \beta l_{1} x_{1} x_{2} .
\end{gathered}
$$

We now present the following result.

Proposition 3. Consider the reduced closed-loop system [equations (36) $-(39)]$ with $k>0, k \neq \alpha$, and $l=\left(\begin{array}{lll}l_{1} & l_{2} & l_{3}\end{array}\right)$ selected such that the matrix $A_{1}$ given by equation (32) is a Hurwitz matrix. Let $\left(x_{10}, x_{20}, x_{30}, x_{40}\right)$ denote an initial condition with $x_{10} \neq 0$. Then the following hold.

(i) The trajectory $\left(x_{1}(t), x_{2}(t), x_{3}(t), x_{4}(t)\right)$ is bounded for all $t \geq 0$ and converges exponentially to zero.

(ii) The control $\left(v_{1}(t), v_{2}(t)\right)$ is bounded for all $t \geq 0$ and converges exponentially to zero.

Proof. Consider the reduced closed-loop system [equations (36)-(39)] and let $\left(x_{10}, x_{20}, x_{30}, x_{40}\right)$ denote an initial condition with $x_{10} \neq 0$

(i) We have $x_{1}(t)=x_{10} \mathrm{e}^{-k t}$. Since $x_{10} \neq 0, \quad x_{1}(t) \neq 0$, $\forall t \in[0, \infty)$. Under the stated assumptions, the reduced closed-loop system in the transformed coordinates is globally 
exponentially stable. Thus, $\left(x_{1}(t), x_{2}(t), x_{3}(t) / x_{1}(t), x_{4}(t) / x_{1}(t)\right)$ converges exponentially to zero. Hence, the trajectory $\left(x_{1}(t), x_{2}(t), x_{3}(t), x_{4}(t)\right)$ is bounded for all $t \geq 0$ and converges exponentially to zern.

(ii) From the above discussion, each term in the control $v$ given by equations (34) and (35) consists of bounded terms which converge exponentially to zero. Hence, the result follows.

Remark 4. The above result demonstrates that for initial conditions satisfying $x_{10} \neq 0$, the feedback control law [equations (34) and (35)] is well defined for all $t \geq 0$. Moreover, it drives the system [equations (36)-(39)] to the origin, while avoiding the set

$$
N=\left\{\left(x_{1}, x_{2}, x_{3}, x_{4}\right) \mid x_{1}=0,\left(x_{1}, x_{2}, x_{3}, x_{4}\right) \neq 0\right\} .
$$

Note that one can use a finite-time feedback control law (Haimo, 1986) to move the system away from $N$.

4.2. Stabilization of the complete system. We now return to the problem of asymptotic stabilization of the system [equations (8)-(13)] with $u_{1}$ and $u_{2}$, instead of $x_{5}$ and $x_{6}$, as control inputs. Since $u_{1}=\dot{x}_{5}$ and $u_{2}=\dot{x}_{6}$, the problem corresponds to the classical situation where integrators are added at the input level. It should be remarked that the integrator back-stepping approach developed for smooth systems (Krstić et al., 1995) cannot be directly applied here due to the discontinuous nature of the system.

Again restrict consideration to $x_{1} \neq 0$ and consider the following controller:

$$
\begin{aligned}
& u_{1}(x)=-K\left(x_{5}-v_{1}\left(x_{1}, x_{2}, x_{3}, x_{4}\right)\right)+s_{1}(x), \\
& u_{2}(x)=-L\left(x_{6}-v_{2}\left(x_{1}, x_{2}, x_{3}, x_{4}\right)\right)+s_{2}(x),
\end{aligned}
$$

where $v_{1}\left(x_{1}, x_{2}, x_{3}, x_{4}\right)$ and $v_{2}\left(x_{1}, x_{2}, x_{3}, x_{4}\right)$ denote the feedback controls [equations (34) and (35)] for the reduced system; and $s_{1}(x)$ and $s_{2}(x)$ correspond to their time derivatives along the trajectories of the system [equations (8)-(13)]

$$
\begin{gathered}
s_{1}(x)=-k x_{5}, \\
s_{2}(x)=-l_{1}\left(x_{6}+x_{3} x_{5}\right)-l_{2} \frac{x_{4}-x_{2} x_{5}}{x_{1}}+l_{3} \frac{\alpha x_{4}+\beta x_{5} x_{6}}{x_{1}} \\
+l_{2} \frac{x_{3} x_{5}}{x_{1}^{2}}+l_{3} \frac{x_{4} x_{5}}{x_{1}^{2}} .
\end{gathered}
$$

Now assume that the control parameters are selected such that $k, l_{1}, l_{2}$ and $l_{3}$ satisfy the conditions of Proposition 3 and $K>k, L>0$. The main idea behind the proposed control law is to implement the control law [equations (34) and (35)] through the integrators by choosing the gains $K$ and $L$ appropriately, while avoiding the set

$$
N^{\prime}=\left\{x \in \mathbf{M} \mid x_{1}=0, x \neq 0\right\} .
$$

Consider the coordinate transformation

$$
\begin{gathered}
y=x_{1}, \quad z_{1}=x_{2}, \quad z_{2}=\frac{x_{3}}{x_{1}}, \quad z_{3}=\frac{x_{4}}{x_{1}}, \\
w_{1}=x_{5}+k x_{1}, \quad w_{2}=x_{6}+l_{1} x_{2}+l_{2} \frac{x_{3}}{x_{1}}+l_{3} \frac{x_{4}}{x_{1}} .
\end{gathered}
$$

It can be shown that in the above coordinates the closed-loop system can be written as

$$
\begin{gathered}
\dot{y}=-k y+w_{1}, \\
\dot{z}=\left(A_{1}+A_{2}(t)\right) z+h(t), \\
\dot{w}_{1}=-K w_{1}, \\
\dot{w}_{2}=-L w_{2},
\end{gathered}
$$

where $A_{1}$ is the matrix given by equation (32) and

$$
\begin{aligned}
\tilde{A}_{2}(t)= & \left(\begin{array}{ccc}
0 & r_{1}(t) & 0 \\
-r_{2}(t) & r_{2}(t) & 0 \\
\beta l_{1} r_{2}(t) & \beta l_{2} r_{2}(t) & \left(\beta l_{3}-1\right) r_{2}(t)
\end{array}\right), \\
r_{1}(t)= & \left(\mathrm{e}^{-k t} y_{0}+\frac{\mathrm{e}^{-k t}-\mathrm{e}^{-K t}}{K-k} w_{10}\right) \\
& \times\left(-k \mathrm{e}^{-k t} y_{0}+\frac{k \mathrm{e}^{-k t}-K \mathrm{e}^{-K t}}{k-K} w_{10}\right), \\
r_{2}(t)= & \left(\mathrm{e}^{-k t} y_{0}+\frac{\mathrm{e}^{-k t}-\mathrm{e}^{-K t}}{K-k} w_{10}\right)-\mathrm{e}^{-K_{t} w_{10}}, \\
h(t)= & \left(\begin{array}{lll}
1 & 0 & \beta\left(k-r_{2}(t)\right)^{\mathrm{T}} \mathrm{e}^{-L_{1}} w_{20} .
\end{array}\right.
\end{aligned}
$$

The $\left(y, w_{1}, w_{2}\right)$-dynamics is globally exponentially stable at $\left(y, w_{1}, w_{2}\right)=(0,0,0)$. Moreover, it can be easily shown that if $y_{0} w_{10} \geq 0$ (or, equivalently, $x_{10}\left(x_{50}+k x_{10}\right) \geq 0$ ), then $\tilde{A}_{2}(t)$ and $h(t)$ go to zero as $t \rightarrow \infty$ and

$$
\int_{0}^{\infty}\left\|A_{2}(t)\right\| \mathrm{d} t<\infty, \quad \int_{0}^{\infty}\|h(t)\| \mathrm{d} t<\infty .
$$

Thus, for any initial condition $\left(y_{0}, z_{0}, w_{10}, w_{20}\right)$ satisfying $y_{0} \neq 0$ and $y_{0} w_{10} \geq 0$, both the trajectory $\left(y(t), z(t), w_{1}(t), w_{2}(t)\right)$ and the control $\left(u_{1}(t), u_{2}(t)\right)$ are bounded for all $t \geq 0$ and converge exponentially to zero.

We now present the following result.

Proposition 4. Consider the system [equations (8)-(13)] with the feedback controls (40) and (41), where the control parameters are selected such that $k, l_{1}, l_{2}$ and $l_{3}$ satisfy the conditions of Proposition 3 and $K>k, L>0$. Let $\left(x_{10}, x_{20}, x_{30}, x_{40}\right.$, $\left.x_{50}, x_{60}\right)$ denote an initial condition satisfying $x_{10} \neq 0$ and $x_{10}\left(x_{50}+k x_{10}\right) \geq 0$. Then the following hold.

(i) The trajectory $\left(x_{1}(t), x_{2}(t), x_{3}(t), x_{4}(t), x_{5}(t), x_{6}(t)\right)$ is bounded for all $t \geq 0$ and converges exponentially to zero.

(ii) The control $\left(u_{1}(t), u_{2}(t)\right)$ is bounded for all $t \geq 0$ and converges exponentially to zero.

Remark 5. Note that the above choice of the feedback control guarantees that

$$
x_{1}(t)=\mathrm{e}^{-k t} x_{10}+\frac{\mathrm{e}^{-k t}-\mathrm{e}^{-K t}}{K-k}\left(x_{50}+k x_{10}\right) .
$$

It can be easily seen that if $x_{10} \neq 0$ and $x_{10}\left(x_{50}+k x_{10}\right) \geq 0$, then $x_{1}(t) \neq 0, \forall t \in,[0, \infty)$. Thus, for all initial conditions satisfying $x_{10} \neq 0$ and $x_{10}\left(x_{50}+k x_{10}\right) \geq 0$, the feedback control law [equations (40) and (41)] is well defined for all $t \geq 0$. Moreover, it drives the system [equations $(8)-(13)]$ to the origin, while avoiding the set [equation (42)]. Clearly, one can use a finitetime feedback control law to move the system to a state satisfying the conditions of Proposition 4, e.g.

$$
\begin{gathered}
u_{1}=-\left|x_{1}-\varepsilon\right|^{a} \operatorname{sign}\left(x_{1}-\varepsilon\right)-\left|x_{3}\right|^{b} \operatorname{sign}\left(x_{5}\right), \\
u_{2}=0,
\end{gathered}
$$

where $b \in(0,1), a>b /(2-b)$ and $\varepsilon \neq 0$ are constants, can be used to transfer the system to a state satisfying the conditions of Proposition 4 in finite time.

\section{Example}

We illustrate the results of the paper with a simulation example of a surface-vessel model with two independent propellers as shown in Fig. 1. The model parameters are given by

$$
\begin{gathered}
\mathrm{m}_{11}=200 \mathrm{~kg}, \quad m_{22}=250 \mathrm{~kg}, \quad m_{33}=80 \mathrm{~kg} \mathrm{~m}^{2}, \\
d_{11}=70 \mathrm{~kg} \mathrm{~s}^{-1}, \quad d_{22}=100 \mathrm{~kg} \mathrm{~s}^{-1}, \quad d_{33}=50 \mathrm{~kg} \mathrm{~m}^{2} \mathrm{~s}^{-1} .
\end{gathered}
$$


A computer implementation of the discontinuous feedback control law specified in Proposition 4 was used to asymptotically stabilize the origin. The results of the simulation for a sample initial condition given by $x_{0}=(0,1,1,0,1,0)$ (or equivalently $\left.\left(x, y, \psi, v_{x}, v_{y}, \omega_{z}\right)=(1,1,0,0,0,1)\right)$ are shown in Figs. $2-5$. Note that this initial condition does not satisfy the conditions of Proposition 4. Thus, the controls given by equations (47) and (48) with $\varepsilon=0.5$ and $a=b=\frac{1}{3}$ were first used to move the system to a state satisfying the conditions of Proposition 4 .
Then, at $t=5.25 \mathrm{~s}$, the controls were switched to those specified in Proposition 4 with

$$
k=0.5, \quad l=(5.00,2.50,-7.25), \quad K=1, \quad L=1 .
$$

Note that the above choice of the gain matrix $l$ locates the eigenvalues of the matrix $A_{1}$ given by equation (32) at $(-0.5,-0.5,-0.5)$. Figure 2 shows the time responses of the configuration variables $x, y$ and $\psi$. The time responses for the

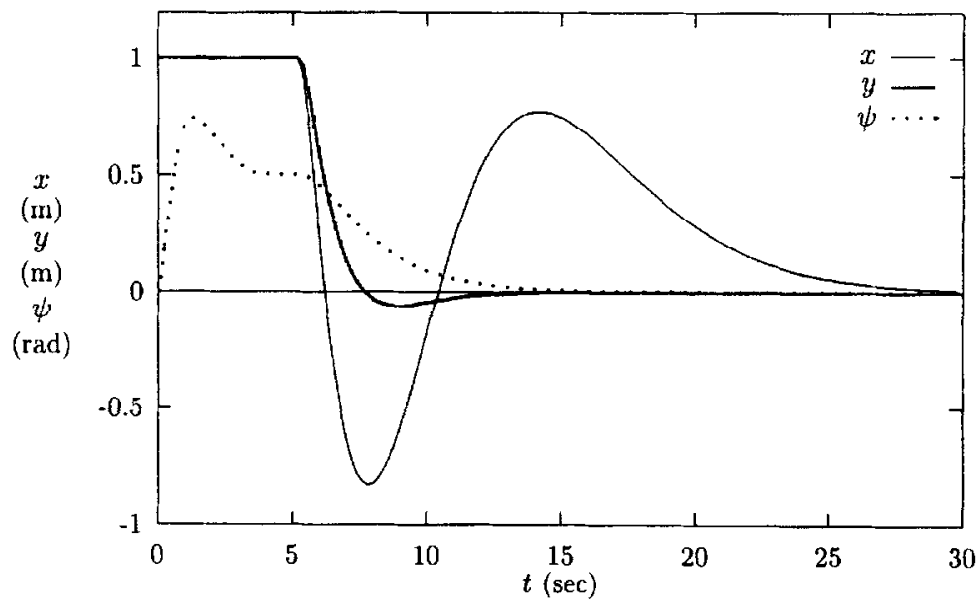

Fig. 2. Time responses for configuration variables.

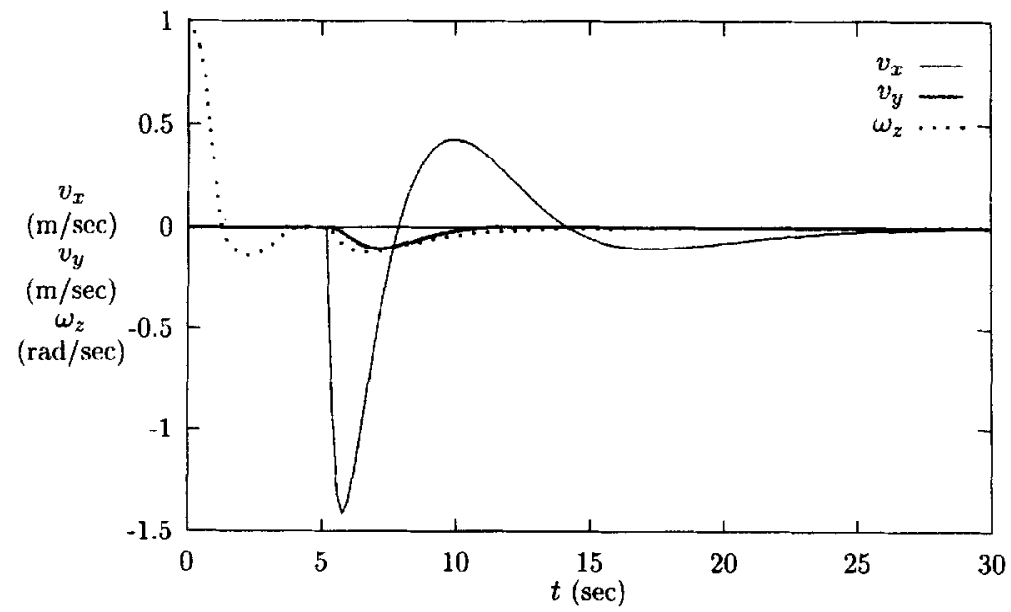

Fig. 3. Time responses for velocity variables.



Fig. 4. Controls $F_{x}$ and $T_{z}$. 


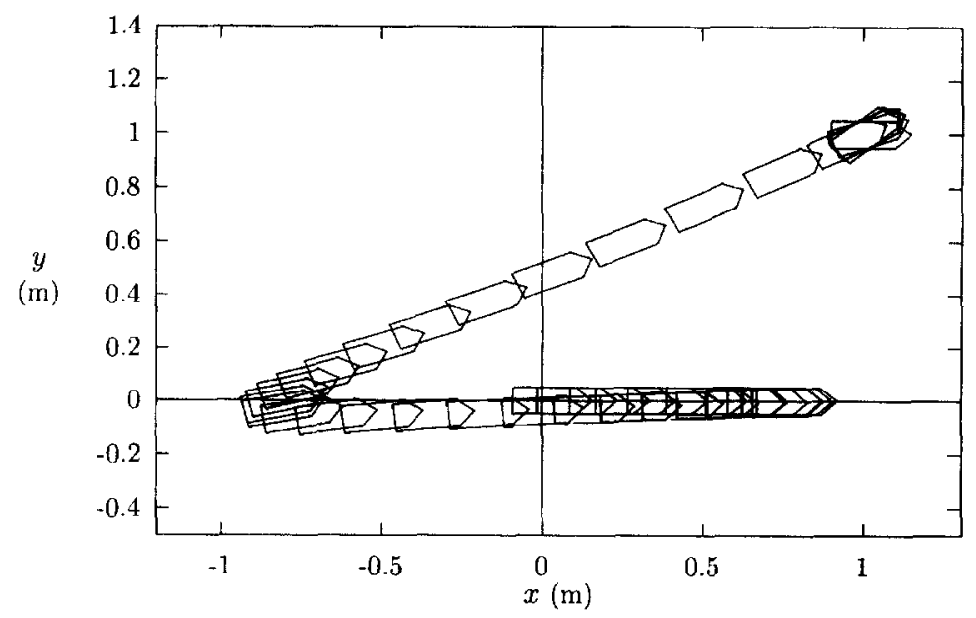

Fig. 5. Surface vessel configuration.

velocities $v_{x}, v_{y}$ and $\omega_{z}$ and the controls $F_{x}$ and $T_{z}$ are shown in Figs. 3 and 4, respectively. Exponential convergence of the closed-loop state and control trajectories can be observed. Figure 5 illustrates the configuration of the surface vessel for a sequence of time instants. The motion until the control switching occurs is a pure yaw motion, which changes the orientation of the vehicle from $\psi=0$ to 0.5 while keeping the I-frame position of the vehicle constant. After the control switching, the vehicle approaches the origin with monotonically decreasing $\psi$.

Remark 6. The simulation results indicate that, with the proposed control law, a significant amount of control effort may be required at certain stages of the motion. A modification of this control law is certainly essential to account for both saturation constraints and rate limits that may be imposed on the control inputs. Such a modification seems to be possible using the ideas introduced in Tsiotras and Luo (1996).

\section{Conclusions}

Following a controllability and stabilizability analysis, timeinvariant discontinuous feedback control laws have been derived for the asymptotic stabilization of a surface vessel with two independent thrusters. Boundedness and convergence of the closed-loop state and control trajectories have been demonstrated. The effectiveness of the proposed feedback control laws has been illustrated through a simulation example.

In this paper, a number of assumptions have been made about the surface vessel dynamics to simplify the presentation of the results. Future research in this area includes relaxation of certain of these assumptions as mentioned in Remark 2.

Acknowledgements--The author wishes to acknowledge the support provided by the Dutch Institute of Systems and Control that enabled his visit to the University of Twente during the academic year $1995-1996$

\section{References}

Arnold, V. I. (1983). Geometrical Methods in the Theory of Ordinary Differential Equations. Springer, New York.

Astolfi, A. (1995). Exponential stabilization of nonholonomic systems via discontinuous control. In Proc. IF AC Nonlincar Control System Design Symp. (NOLCOS), Tahoe City, pp. 741-746.

Bloch, A. M., M. Reyhanoglu and N. H. McClamroch (1992). Control and stabilization of nonholonomic dynamic systems. IEEE Trans. Automat. Control, AC-37, 1746-1757.

Brocketl, R. W. (1983). Asymptotic stability and feedback stabilization. In Differential Geometric Control Theory, Brockett R. W., R. S. Millman and H. J. Sussmann, eds, pp. 181-191. Birkhauser, Boston
Coron, J.-M. and E.-Y. Kerai (1996). Explicit feedbacks stabilizing the attitude of a rigid spacecraft with two control torques. Automatica, 32, 669-677.

Egeland, O., M. Dalsmo and O. J. Sordalen (1996). Feedback control of a nonholonomic underwater vehicle with constant desired configuration. Int. J. Robotics Res., to appear.

Fossen, T. I. (1994). Guidance and Control of Ocean Vehicles. Wiley, New York.

Haimo, V. T. (1986). Finite time controllers. SIAM J. Control Optim., 24, 760-770.

Krstić, M., I. Kanellakopoulos and P. Kokotovic (1995). Nonlinear and Adaptive Control. Wiley, New York.

Leonard, N. E. (1995). Control synthesis and adaptation for an underactuated autonomous underwater vehicle. IEEE $J$. Oceanic Engng, 20, 211-220.

Nijmeijer, H. and A. J. van der Schaft (1990). Nonlinear Dynamical Control Systems. Springer, New York.

Oriolo, G. and Y. Nakamura (1991). Control of mechanical systems with second-order nonholonomic constraints: underactuated manipulators. In Proc. IEEE Conf. on Decision and Control, Brighton, U.K., pp. 2398-2403.

Reyhanoglu, M. (1996). Control and stabilization of a surface vessel. In Proc. IEEE Conf. on Decision and Control, Kobe, Japan, pp. 2371-2376.

Reyhanoglu, M. and N. H. McClamroch (1992). Planar reorientation maneuvers of space multibody systems using internal controls. J. Guidance Control Dyn., 15, 1475-1480.

Reyhanoglu, M., A. J. van der Schaft, N. H. McClamroch and I. Kolmanovsky (1996). Dynamics and control of a class of underactuated mechanical systems. In Proc. IEEE Conf. on Decision and Control, Kobe, Japan, pp. $1682-1687$.

Slotine, J.-J. E. and W. Li (1991). Applied Nonlinear Control. Prentice-Hall, Englewood Cliffs, NJ.

Spong, M. W. (1996). Energy based control of a class of underactuated mechanical systems. In Proc. IFAC World Cong., San Francisco, CA, pp. 431-435.

Sussmann, H. J. (1979). Subanalytic sets and feedback control. J. Differential Equations, 31, 31-52.

Sussmann, H. J. (1987). A general theorem on local controllability. SIAM J. Control Optim., 25(1), 158-194.

Tsiotras, P. and J. Luo (1996). A reduced-effort control law for underactuated rigid bodies. In Proc. IEEE Conf. on Decision and Control, Kobc, Japan, pp. 495-496.

Wichlund, K. Y., O. J. Sordalen and O. Egeland (1995). Control of vehicles with second-order nonholonomic constraints: underactuated vehicles. In Proc. Eur. Control Conf., Rome, Italy, pp. 3086-3091.

Zabczyk, J. (1989). Some Comments on Stabilizability. Appl. Math. Optim., 19, pp. $1-9$. 